Vol. 44 (1991) [303-324]

\title{
FINITE ALGEBRAS THAT GENERATE AN INJECTIVELY COMPLETE MODULAR VARIETY
}

\author{
Keith A. Kearnes
}

\begin{abstract}
We extend Kollár's result on finitely generated, injectively complete congruence distributive varieties to the congruence modular setting. By doing so we show that, given any finite algebra $\mathbf{A}$ of finite type, there is an algorithm to decide whether $\mathcal{V}(\mathbf{A})$ is an injectively complete, congruence modular variety.
\end{abstract}

\section{INTRODUCTION}

Many authors have considered the question of which finite algebras $\mathbf{A}$ generate an injectively complete variety, that is, a variety in which every member can be embedded into an injective member. It is known that a variety $\mathcal{V}$ is injectively complete, or has enough injectives, if and only if it is residually small, has the congruence extension property and has the amalgamation property. This follows from a combination of results due to Banascheski [2] and Taylor [17]. Day, in [5], proved that every primal algebra generates an injectively complete variety. Quackenbush extended this result in [16] by proving that if $\mathbf{A}$ is quasiprimal then $\mathcal{V}(\mathbf{A})$ has $\mathbf{A P}$ (equivalently, is injectively complete) if and only if $\mathbf{A}$ is demi-semi-primal. The characterisation of finitely generated injectively complete varieties was further extended to congruence distributive varieties by Kollár [12]. We extend Kollár's theorem to the modular setting with the following result:

ThEOREM 1.1. Let $\mathcal{V}$ be a finitely generated, congruence modular variety. Let $\mathcal{K}=\operatorname{HSSI}(\mathcal{V})$. Then $\mathcal{V}$ has enough injectives if and only if:

(a) $\mathcal{K} \models_{\text {con }} \mathrm{C} 2+\mathrm{R}$,

(b) each maximal SI is *-injective over $\mathcal{K}$ and

(c) Every retract of a maximmal SI is isomorphic to a direct product of SI algebras that are ${ }^{*}$-injective over $\mathcal{K}$.

Here $\operatorname{SI}(\mathcal{V})$ denotes the class of subdirectly irreducible algebras in $\mathcal{V}$. A maximal $\mathrm{SI}$ is one that is maximal under embeddability in $\operatorname{SI}(\mathcal{V})$.

Received 6 November 1990

Copyright Clearance Centre, Inc. Serial-fee code: 0004-9729/91 \$A2.00+0.00. 
Theorem 1.1 yields an effective algorithm for determining if a finite algebra of finite type generates an injectively complete modular variety. This is because the condition that $\mathcal{K} \models_{\text {con }} \mathrm{C} 2+\mathrm{R}$ can be effectively verified when $\mathcal{V}$ is of finite type and a finite algebra generating $\mathcal{V}$ is known. If this condition holds then $\mathcal{K}$ is a finite category. Further, it is possible to give a bound on the cardinality and the number of isomorphism types of algebras in $\mathcal{K}$ in terms of the cardinality of the algebras that generate $\mathcal{V}$.

Our notation for algebras is fairly standard and the reader is referred to [14]. For the notation and basic results of modular commutator theory the reader is referred to [7].

An object $\mathrm{I}$ in a category $\mathcal{K}$ is injective over $\mathcal{K}$ if whenever we have a diagram in $\mathcal{K}:$

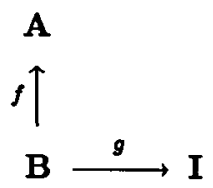

where $f$ is a monic arrow and $g$ is arbitrary, then there is an arrow $\widehat{g}: \mathbf{A} \rightarrow \mathbf{I}$ satisfying $\widehat{g} \circ f=g$. When we consider a variety as a category we choose the morphisms to be

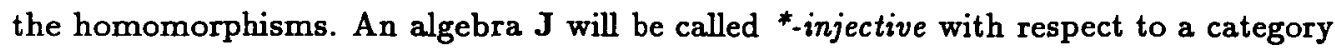
of algebras if the same property holds for $\mathbf{J}$ except the condition that $f$ is monic is replaced by the condition that $f$ is $1-1$. In a variety $\mathcal{V}$ the monic morphisms are precisely the 1-1 homomorphisms, so these two definitions agree for varieties. In fact, this holds for any full subcategory of $\mathcal{V}$ that contains the relatively free algebra $F_{\mathcal{V}}(1)$. However, for an arbitrary (full) subcategory of $\mathcal{V}$, say $\operatorname{HS~SI}(\mathcal{V}),{ }^{*}$-injectivity is weaker than injectivity. In general, we can say that if $I$ is injective over $\mathcal{V}$ then $I$ is *-injective over any full subcategory of $\mathcal{V}$ that contains $I$.

A subalgebra $\mathbf{A}$ of $\mathbf{B}$ is a retract of $\mathbf{B}$ if it is the image of an idempotent endomorphism of $\mathbf{B} . \mathbf{A} \varepsilon \mathcal{V}$ is an absolute retract if it is a retract of any of its extensions in $\nu$.

A variety of algebras $\mathcal{V}$ is said to have the amalgamation property if whenever we have embeddings $f_{1}: \mathbf{A} \rightarrow \mathbf{B}$ and $g_{1}: \mathbf{A} \rightarrow \mathbf{C}$ we can find an algebra $\mathbf{D}$ and embeddings $f_{2}, g_{2}$ which complete a commutative diagram:

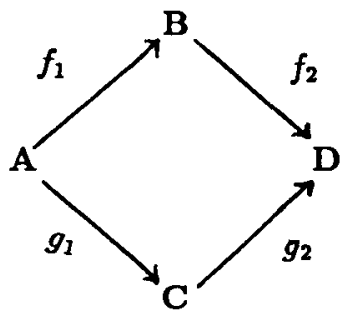


In this case we will say that the triple $\left(f_{2} ; g_{2} ; \mathbf{D}\right)$ completes the amalgam, $\left(\mathbf{A} ; f_{1}, \mathbf{B} ; g_{1}, \mathbf{C}\right) . \mathcal{V}$ is residually small just in case it has a bound on the size of its subdirectly irreducible members. $\mathcal{V}$ has the congruence extension property if for every embedding $f: \mathbf{B} \rightarrow \mathbf{A}$ and every congruence $\theta \varepsilon$ Con $\mathbf{B}$ there is a congruence $\psi \varepsilon$ Con A such that $\theta=\left.\psi\right|_{\mathbf{B}}=f^{-1}(\psi)$.

We adhere to the custom of excluding the empty algebra from membership in a variety $\mathcal{V}$. When investigating injective completeness some authors prefer to consider $\mathcal{V} \cup\{\phi\}$ when $\mathcal{V}$ has no nullary operations. We will write $\operatorname{Mod} \Sigma(\mathcal{V})$ for $\mathcal{V}$ if $\mathcal{V}$ has nullary operations or for $\mathcal{V} \cup\{\phi\}$ otherwise. (The morphisms for $\mathcal{V} \cup\{\phi\}$ are just all homomorphisms and empty maps.) Let us compare these two situations in the next theorem. We omit the easy proof.

THEOREM 1.2. If $\phi \varepsilon \operatorname{Mod} \Sigma(\mathcal{V})$ then

(a) $\operatorname{Mod} \Sigma(\mathcal{V})$ is injectively complete if and only if $\mathcal{V}$ is injectively complete and every $\mathbf{A} \varepsilon \mathcal{V}$ can be embedded into an algebra in $\mathcal{V}$ that has a 1element subalgebra.

If $\mathcal{V}$ is locally finite then we can rewrite this as:

$\left(a^{\prime}\right) \operatorname{Mod} \Sigma(\mathcal{V})$ is injectively complete if and only if $\mathcal{V}$ is injectively complete and every maximal SI has a 1-element subalgebra.

In either case, the injective algebras in $\operatorname{Mod} \Sigma(\mathcal{V})$ are precisely the $\mathcal{V}$-injectives that have a 1-element subalgebra.

(b) $\operatorname{Mod} \Sigma(\mathcal{V})$ has the AP if and only if $\mathcal{V}$ has the AP and given any two algebras in $\mathcal{V}$ there exists a third that jointly embeds them.

(c) $\operatorname{Mod} \Sigma(\mathcal{V})$ is residually small if and only if $\mathcal{V}$ is.

(d) $\operatorname{Mod} \Sigma(\mathcal{V})$ has the CEP if and only if $\mathcal{V}$ does.

Using 1.2 the reader can easily adjust the hypotheses of any of our results if he prefers to consider $\operatorname{Mod} \Sigma(\mathcal{V})$ instead of $\mathcal{V}$.

\section{Locally Finite, Injectively Complete Varieties}

THEOREM 2.1. Let $\mathcal{V}$ be a locally finite, residually small variety with the CEP. Then $\mathcal{V}$ has only finitely many nonisomorphic SI's, all of which are finite. In particular, $\mathcal{V}$ is finitely generated.

Proof: By [1], any locally finite RS + CEP variety is residually less than $n$ for some finite $n$. Hence all members of $\operatorname{SI}(\mathcal{V})$ are homomorphic images of the (finite) free algebra $F_{\mathcal{V}}(n)$.

This means that determining the locally finite, injectively complete varieties reduces to the question of which finite algebras generate an injectively complete variety. 
In this paper we focus on finite algebras that generate a congruence modular variety. However, it is our expectation that analogous results hold for all finite algebras. At least, we believe that given a finite similarity type $\rho$ it is possible to recursively enumerate the finite algebras of type $\rho$ which generate an injectively complete variety. So, for this section, we prove results which do not require the assumption of congruence modularity.

In the next theorem we will let $\mathrm{SI}_{n}(\mathcal{V})$ denote the set of $n$-generated subdirectly irreducible algebras in $\mathcal{V}$ and let $\mathbf{S I}_{\omega}(\mathcal{V})$ denote the set of finitely generated SIs.

THEOREM 2.2. Let $\mathcal{V}$ be a variety that has the CEP. If $\operatorname{SI}_{n}(\mathcal{V})=\operatorname{SI}_{n+1}(\mathcal{V})$ for some finite $n \geqslant 1$, then $\operatorname{SI}_{n}(\mathcal{V})=\operatorname{SI}_{\omega}(\mathcal{V})$. In addition, if $\mathbf{F}_{\mathcal{V}}(n)$ is finite, then $\mathrm{SI}_{n}(\mathcal{V})=\operatorname{SI}(\mathcal{V})$.

Proof: For the first statement, assume the contrary. Then there is a k-generated algebra $\mathrm{A}_{\varepsilon} \operatorname{SI}_{\omega}(\mathcal{V})$ which is not n-generated. Suppose that $\left\{g_{1}, g_{2}, \ldots, g_{k}\right\}$ generates $\mathbf{A}$ and that $\mu=\theta(\mathrm{a}, \mathrm{b})$ is the monolith of $\mathbf{A}$. Define a sequence of subalgebras of $\mathbf{A}$ :

$$
\mathbf{B}_{i}=<a, b, g_{1}, \ldots, g_{i}>, i=n-1, n, \ldots, k .
$$

Since $\left.(\mathrm{a}, \mathrm{b}) \varepsilon \mu\right|_{\mathbf{B}_{i}}$ the inclusion of $\mathbf{B}_{\boldsymbol{i}}$ into $\mathbf{A}$ is essential. $\mathcal{V}$ has the CEP so each $\mathbf{B}_{\boldsymbol{i}}$ must be subdirectly irreducible. $B_{n-1}$ is $(n+1)$-generated so by hypothesis we can find an n-element set of generators. $\mathbf{B}_{i+1}$ is generated by $\mathbf{B}_{i} \cup\left\{g_{i+1}\right\}$ so inductively we conclude that all $\mathbf{B}_{i}$ are n-generated. However, $\mathbf{B}_{k}=\mathbf{A}$ contradicting our assumption that $\mathbf{A}$ is not n-generated.

For the second statement let $\mathbf{C} \varepsilon \operatorname{SI}(\mathcal{V})$. We can find a maximal non-trivial, finitely generated, essential subalgebra of $\mathbf{C}$. This is because all finitely generated essential subalgebras of $\mathbf{C}$ are in $\mathbf{S I}_{\omega}(\mathcal{V})$, so by the preceding paragraph they are homomorphic images of the finite algebra $F_{\mathcal{V}}(n)$. Call such a subalgebra $D$. If $x \varepsilon \mathbf{C} \backslash \mathbf{D}$ then $<$ $\mathbf{D},\{x\}>$ is a non-trivial, finitely generated, essential subalgebra of $\mathbf{C}$ that properly contains $\mathbf{D}$. Hence $\mathbf{D}=\mathbf{C}$ and $\mathbf{C}$ must be finitely generated. By the previous paragraph $\mathrm{C}$ can be generated by $\leqslant n$ elements.

Suppose that for a given finite similarity type $\rho$ we have an algorithm to determine if a finite algebra of type $\rho$ generates a variety with the CEP. Then we can recursively enumerate the finite algebras of type $\rho$ that generate a CEP+RS variety. Here is the procedure we use. At the $n^{\text {th }}$ step in our procedure we compute all isomorphism types of $n$-element algebras of type $\rho$ (there are finitely many) and we use the algorithm to eliminate those which do not generate a CEP variety. Then, for each $\mathbf{A}$ of cardinality $\leqslant n$ about which we are still undecided, we compute the (finite number of) isomorphism types in $\operatorname{SI}_{1}(\mathcal{V}(\mathbf{A})), \ldots, \mathrm{SI}_{n}(\mathcal{V}(\mathbf{A}))$ and check if $\mathrm{SI}_{m}(\mathcal{V}(\mathbf{A}))=\mathrm{SI}_{m+1}(\mathcal{V}(\mathbf{A}))$ for some $m<n$. If this happens, then $A$ belongs on our list of finite algebras of type $\rho$ that 
generate a $\mathrm{CEP}+\mathrm{RS}$ variety. If not, then we remain undecided about $\mathbf{A}$. This finishes the $n^{\text {th }}$ step.

Now we begin looking at the amalgamation property.

LEMMA 2.3. Assume that $\mathcal{V}$ has the CEP. Then $\mathcal{V}$ has the amalgamation property if and only if we can complete each amalgam $\left(\mathbf{A} ; f_{1}, \mathbf{B} ; g_{1}, \mathbf{C}\right)$, where $\mathbf{B}$ is $\mathrm{SI}$ and $g_{1}$ is essential, with a triple $\left(f_{2} ; g_{2} ; \mathbf{D}\right)$ where $\mathbf{D}$ is $\mathrm{SI}$ and $f_{2}$ is essential.

If we can complete all the amalgams $\left(\mathbf{A} ; f_{1}, \mathbf{B} ; g_{1}, \mathbf{C}\right)$ where all algebras are SI and both maps are essential, then we can complete all amalgams in $\mathcal{V}$ where both maps are essential.

Proof: Suppose that we have an arbitrary amalgam $\left(A ; f_{1}, B ; g_{1}, C\right)$. Let $\mathrm{B} \leqslant \Pi \mathrm{B} / \theta_{i}$ be a representation of $\mathrm{B}$ as a subdirect product of subdirectly irreducible algebras and let $\theta_{i}^{\mathbf{A}}=\left.\theta_{i}\right|_{\mathbf{A}}, \theta_{i}^{\mathbf{C}}=a$ maximal congruence $\theta \varepsilon$ Con $\mathbf{C}$ such that $\left.\theta\right|_{\mathbf{A}}=\theta_{\boldsymbol{i}}^{\mathbf{A}}$ (there is at least one since $\mathcal{V}$ has the CEP). If we can amalgamate each $\left(\mathbf{A} / \theta_{i}^{\mathbf{A}} ; f_{1}^{(i)}, \mathbf{B} / \theta_{i} ; g_{1}^{(i)}, \mathbf{C} / \theta_{i}^{\mathbf{C}}\right)$ then the product of the amalgamating algebras, $\left\{\mathbf{D}_{i} \mid i \varepsilon i\right\}$, amalgamates $\mathbf{B}$ with $\mathbf{C} /\left(\cap \theta_{i}^{\mathbf{C}}\right)$ over $\mathbf{A}$. Reversing the roles of $\mathbf{B}$ and $\mathbf{C}$ and taking the product again completes the original amalgam. Hence, all amalgams can be completed if and only if we can complete those where B is SI and C is an essential extension of the algebra $A$.

Consider $\left(\mathbf{A} ; f_{1}, \mathbf{B} ; g_{1}, \mathbf{C}\right)$ where $\mathbf{B}$ is $\mathbf{S I}$ and $g_{1}$ is essential. If $\left(f_{2} ; g_{2} ; \mathbf{D}\right)$ completes this amalgam then we may choose $\psi \varepsilon$ Con $\mathbf{D}$ maximal with respect to the property that $\left.\psi\right|_{\mathbf{B}}=0_{\mathbf{B}}$. Then, $\left.\psi\right|_{\mathbf{A}}=0_{\mathbf{A}}$ and, since $g_{1}$ is essential, $\left.\psi\right|_{\mathbf{C}}=0_{\mathbf{C}}$. Thus $\left.\overline{f_{2}} ; \overline{g_{2}} ; \mathbf{D} / \psi\right)$ completes the amalgam with $\overline{f_{2}}$ essential and (consequently) with $\mathbf{D} / \psi$ subdirectly irreducible.

The argument for the second statement of this lemma is similar to the argument above. Choose a family $\left\{\theta_{i} \mid i \varepsilon I\right\}$ of strictly meet-irreducible congruences that separate the points of $\mathbf{A}$. Let $\boldsymbol{\theta}_{i}^{\mathbf{B}}$ and $\theta_{i}^{\mathbf{C}}$ be maximal extensions of $\theta_{i}$ to $\mathbf{B}$ and $\mathbf{C}$ respectively. Necessarily, these congruences are strictly meet-irreducible and separate the points of $\mathbf{B}$ and $\mathbf{C}$ respectively (that they separate points follows from the fact that $\mathbf{B}$ and $\mathbf{C}$ are essential extensions of $\mathbf{A})$. If all the amalgams $\left(\mathbf{A} / \theta_{i} ; f_{1}^{(i)}, \mathbf{B} / \theta_{i}^{\mathbf{B}} ; g_{1}^{(i)}, \mathbf{C} / \theta_{i}^{\mathbf{C}}\right)$ can be completed, then taking products completes the original amalgam.

Next, we need a generalisation of injective hulls.

Definition 2.4: We say that $\mathbf{S}$ is a shell of $\mathbf{A}$ if $\mathbf{S}$ is an extension of $\mathbf{A}$ (that is, there is a distinguished embedding $\boldsymbol{\xi}: \mathbf{A} \rightarrow \mathbf{S}$ ) and $\mathbf{S}$ and $\boldsymbol{\xi}$ have the following properties:

(a) $\boldsymbol{\xi}: \mathbf{A} \rightarrow \mathbf{S}$ is an essential extension.

(b) There is a set $\left\{\theta_{i} \mid i \varepsilon I\right\}$ of strictly meet-irreducible congruences of $\mathbf{A}$ 
separating the points of $\mathbf{A}$, a set $\left\{\left(f_{i}, \mathbf{B}_{i}\right) \mid i \varepsilon I\right\}$ of maximal essential extensions $f_{i}: \mathbf{A} / \theta_{i} \rightarrow \mathbf{B}_{i}$ and a homomorphism $\lambda: \mathbf{S} \rightarrow \prod_{i \in I} \mathbf{B}_{i}$ such that the following diagram commutes:

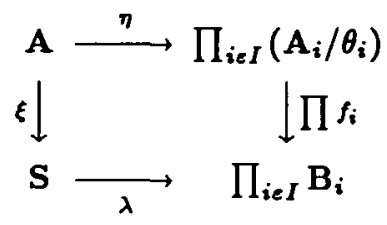

In this diagram $\eta$ denotes the natural map.

(c) $\mathbf{S}$ is maximal with respect to extension among algebras satisfying (a) and (b).

Of course, a given $\mathbf{A} \varepsilon \mathcal{V}$ may have no shell or many nonisomorphic ones. The problem of existence reduces to the question of the existence of the set $\left\{\left(f_{i}, \mathbf{B}_{i}\right) \mid i \varepsilon I\right\}$. For this we need to know that each algebra $A / \theta_{i}$ has a maximal essential extension. However, a result of Taylor in [17] shows that any algebra in a residually small variety does have a maximal essential extension. It follows that if $\mathcal{V}$ is residually small then every algebra $A \in \mathcal{V}$ has at least one shell for every set of strictly meet-irreducible congruences $\left\{\theta_{i} \mid i \varepsilon I\right\}$ which separate the points of $\mathbf{A}$. If $\mathcal{V}$ is a locally finite CEP+RS variety, then $\mathcal{V}$ has only finitely many nonisomorphic subdirectly irreducible algebras and all are finite. One consequence of this is that if $A \varepsilon \mathcal{V}$ is finite, then we may compute a finite bound on the size of the shells of $\mathbf{A}$. This yields a bound on the size of the essential extensions of $\mathbf{A}$. Indeed, we can effectively locate all essential extensions of $\mathbf{A}$ up to isomorphism.

THEOREM 2.5. Let $\mathcal{V}$ be a locally finite CEP+RS variety and let $\mathcal{K}=\operatorname{HSSI}(\mathcal{V})$. The following are equivalent:

(a) $\mathcal{V}$ has enough injectives.

(b) (i) Each maximal SI is *-injective over $\mathcal{K}$ and

(ii) each retract $\mathbf{R}$ of a maximal SI is an absolute retract in $\mathcal{V}$.

(c) (i) Each maximal SI is *-injective over $\mathcal{K}$ and

(ii) Each retract $\mathbf{R}$ of a maximal $\mathrm{SI}$ is a shell for itself with respect to some (in fact, every) set of strictly meet-irreducible congruences on $\mathbf{R}$ that separate the points of $\mathbf{R}$.

Proof: If $\mathcal{V}$ has enough injectives then the class of injectives is precisely the class of absolute retracts in $\mathcal{V}$ and therefore it contains the maximal SIs. Further, the class of injectives is closed under retracts. This shows that (a) implies (b)(ii). To show that (a) implies (b)(i), note that since maximal SIs are absolute retracts in $\mathcal{V}$, they are injective in $\mathcal{V}$ and so they are at least ${ }^{*}$-injective in $\mathcal{K}$. 
To show that (b) implies (c) we only need to verify that (c)(ii) holds. As we have remarked, the fact that $\mathcal{V}$ is $\mathbf{R S}$ implies that every algebra $\mathbf{R}$ in $\mathcal{V}$ has a shell $\mathbf{S}$ which, by definition, is an essential extension of $\mathbf{R}$. If $\mathbf{R}$ is a retract of a maximal $S I$ then (b)(ii) yields that $\mathbf{R}$ is an absolute retract, hence a retract of $\mathbf{S}$. Necessarily $\mathbf{R}=\mathbf{S}$. Thus, the only shell for $\mathbf{R}$ is $\mathbf{R}$ itself. Hence, $\mathbf{R}$ is a shell for itself with respect to any set of congruences which separate the points of $\mathbf{R}$.

We need to show that (c) implies (a). Equivalently, we must show that (c) forces $\mathcal{V}$ to have AP. Let $\left(\mathbf{A} ; f_{1}, \mathbf{B} ; g_{1}, \mathbf{C}\right)$ be an amalgam in $\mathcal{V}$ where all algebras are SI and both maps are essential. Find $f_{2}$ and $\mathbf{D}$ such that $\mathbf{D}$ is a maximal $\mathbf{S I}$ and $f_{2}: \mathbf{B} \rightarrow \mathbf{D}$ is 1-1. This gives us a diagram in $\mathcal{K}$ :

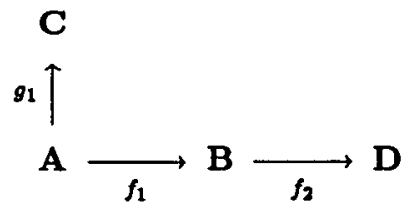

Since $\mathbf{D}$ is *-injective over $\mathcal{K}$ there is a map $g_{2}: \mathbf{C} \rightarrow \mathbf{D}$ where $g_{2} \circ g_{1}=f_{2} \circ f_{1}$. $g_{2}$ is $1-1$ since $g_{1}$ is essential and $f_{2} \circ f_{1}$ is $1-1$. Hence $\left(f_{2} ; g_{2} ; \mathbf{D}\right)$ completes this amalgam. Lemma 2.3 shows that $\mathcal{V}$ admits a limited amount of amalgamation: we can amalgamate diagrams in which both maps are essential.

Now let $\left(\mathbf{A} ; f_{1}, \mathbf{B} ; g_{1}, \mathbf{C}\right)$ be any amalgam where $\mathbf{B}$ is $\mathbf{S I}$ and $g_{1}$ is essential. If we can complete the amalgam $\left(\mathbf{A} ; f \circ f_{1}, \mathbf{B}^{\prime} ; g_{1}, \mathbf{C}\right)$ where $f: \mathbf{B} \rightarrow \mathbf{B}^{\prime}$ is $1-1$, then the amalgamating algebra together with the obvious composed maps will complete the original amalgam. Therefore we may assume from now on that $B$ is a maximal $S I$. Let $\mathbf{A}^{\prime}$ be a maximal essential subextension of $\mathbf{A}$ in $\mathbf{B}$. Say

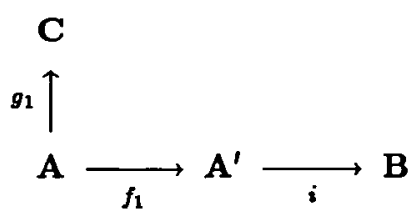

In $\left(\mathbf{A} ; f_{1}, \mathbf{A}^{\prime} ; g_{1}, \mathbf{C}\right)$ both maps are essential so we can find $\left(h_{2} ; g_{2} ; \mathbf{C}^{\prime}\right)$ with both maps essential which completes this amalgam. We have

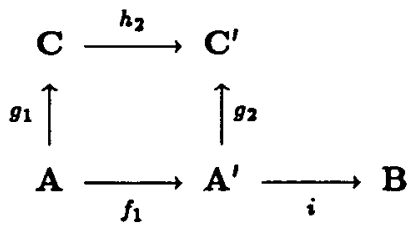

We need to show that $\left(\mathbf{A}^{\prime} ; i, \mathbf{B} ; g_{2}, \mathbf{C}^{\prime}\right)$ can be completed. If we can do this then we will have completed $\left(A ; f_{1}, B ; g_{1}, C\right)$ and the result of Lemma 2.3 implies that $\mathcal{V}$ has AP. This will finish the proof. 
Let $\theta$ be a maximal congruence of $B$ that has the property that $\left.\theta\right|_{A^{\prime}}=0$. We have a diagram in $\mathcal{K}$ :

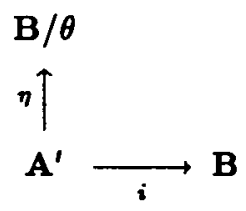

where $\eta$ denotes the restriction to $\mathbf{A}^{\prime}$ of the natural map from $\mathbf{B}$ to $\mathbf{B} / \boldsymbol{\theta}$. Further, $\eta$ is 1-1 and essential. Since $B$ is *-injective over $\mathcal{K}$ and $\eta$ is essential, there exists a 1-1 map $\hat{i}: \mathbf{B} / \theta \rightarrow \mathbf{B}$ such that $\widehat{i} \circ \eta=i$. Hence $\hat{i}(\mathbf{B} / \theta)$ is a subalgebra of $\mathbf{B}$ that is an essential extension of $A^{\prime}$. The choice of $A^{\prime}$ forces $\widehat{i}(B / \theta)=A^{\prime}$. Hence $A^{\prime}$ is a retract of $\mathbf{B}$.

Now let us invoke (c)(ii). $\quad \mathbf{A}^{\prime}$ is a shell for itself with respect to some choice of strictly meet-irreducible congruences that separate the points of $\mathbf{A}^{\prime}$. There is some choice of congruences $\left\{\theta_{i} \mid i \varepsilon I\right\}$ on $A^{\prime}$ and some choice of maximal essential extensions $f_{i}: \mathbf{A}^{\prime} / \theta_{i} \rightarrow \mathbf{D}_{i}$ such that $\nu\left(\mathbf{A}^{\prime}\right)$ is a maximal essential subextension of itself in $\prod_{i \in I} \mathbf{D}_{i}$ where $\nu$ is the natural embedding of $\mathbf{A}^{\prime}$ into $\prod_{i \in I} \mathbf{D}_{i}$. This means that $\nu\left(\mathbf{A}^{\prime}\right)$ has no proper essential subextensions in $\prod_{i \in I} D_{i}$. Let $\theta_{i}^{C^{\prime}}$ be a maximal extension of $\theta_{i}$ to to $\mathbf{C}^{\prime}$. In the amalgam $\left(\mathbf{A}^{\prime} / \theta_{i} ; f_{i}, \mathbf{D}_{i} ; g_{2}^{(i)}, \mathbf{C}^{\prime} / \theta_{i}^{\mathbf{C}^{\prime}}\right)$ all algebras are SI and all maps are essential, so we can complete this amalgam. The algebra that completes this amalgam can be chosen to be an essential extension of both $\mathbf{C}^{\prime} / \theta_{i}^{\mathbf{C}^{\prime}}$ and $\mathbf{D}_{i}$. Since $\mathbf{D}_{i}$ is already maximal with respect to essential extensions, we can complete this amalgam with $\left(i d_{\mathbf{D}_{i}} ; g_{3}^{(i)} ; \mathbf{D}_{i}\right)$ where $g_{3}^{(i)}$ is such that $f_{i}=g_{3}^{(i)} \circ g_{2}^{(i)}: \mathbf{A} / \theta_{i} \rightarrow \mathbf{C}^{\prime} / \theta_{i}^{\mathbf{C}^{\prime}} \rightarrow \mathbf{D}_{i}$ Now, taking products and letting $\gamma$ denote the composed map $\Pi g_{3}^{(i)} \circ \nu^{\prime}: \mathbf{C}^{\prime} \rightarrow$ $\prod_{i \in I} \mathbf{C}^{\prime} / \theta_{i}^{\mathbf{C}^{\prime}} \rightarrow \prod_{i \in I} \mathbf{D}_{i}$, we get that

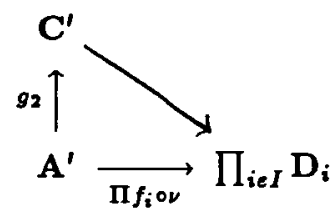

commutes. Since we know that $g_{2}$ an essential embedding, that $\gamma$ is $1-1$ and that $\mathbf{A}^{\prime}$ is a maximal essential subextension of $\prod_{i \in I} D_{i}$ we get that $g_{2}$ is actually an isomorphism. 
Now let us return to our diagram:

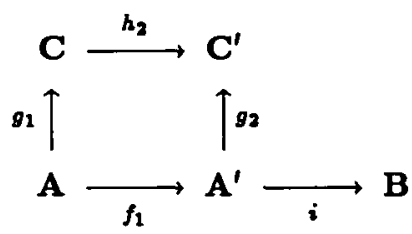

We have just shown that $g_{2}$ is an isomorphism. To complete $\left(\mathbf{A}^{\prime} ; i, \mathbf{B} ; g_{2}, \mathbf{C}^{\prime}\right)$ simply let $h_{3}: \mathbf{C}^{\prime} \rightarrow \mathrm{B}$ be the map $i \circ g_{2}^{-1}$. This shows that the original amalgam $\left(\mathbf{A} ; f_{1}, \mathbf{B} ; g_{1}, \mathbf{C}\right)$ is completed by $\left(i d_{B} ; i \circ g_{2}^{-1} \circ h_{2} ; B\right)$. As we have remarked, this finishes the proof. However, note that in this paragraph we have shown that if $A^{\prime}$ is retract of a maximal SI and $A^{\prime}$ is a shell for itself with respect to some set of congruences, then there is no proper essential extension $\mathbf{C}^{\prime}$ of $\mathbf{A}^{\prime}$. Hence $\mathbf{A}^{\prime}$ is the only shell for itself and it is an absolute retract in $\mathcal{V}$. This explains our wording of condition (c)(ii): if $\mathcal{V}$ is a locally finite, CEP +RS variety for which (c)(i) holds, then a retract of a maximal $S I$ is a shell for itself with respect to some set of congruences that separate points if and only if it is a shell for itself with respect to any set of congruences that separate points.

Again suppose that for a given finite similarity type $\rho$ we have an algorithm for determining if a finite algebra of type $\rho$ generates a variety with the CEP. Then we can recursively enumerate the finite algebras of type $\rho$ that generate a variety with $\mathrm{AP}+\mathrm{RS}+\mathrm{CEP}$, that is, an injectively complete variety. We simply use the procedure described earlier for enumerating the algebras that generate a variety with RS+CEP and we check for AP along the way. That is, at the end of the $n^{\text {th }}$ step in our earlier algorithm we isolate those algebras which have just been shown to generate varieties with $\mathrm{RS}+\mathrm{CEP}$. We have already computed $\operatorname{SI}(\mathcal{V}(\mathbf{A}))$ for these algebras so we can test for AP by using Theorem 2.5(c). If we reach this stage, then $\mathcal{K}$ has only finitely many (nonisomorphic) algebras and all are finite. Checking 2.5(c) will only require a finite amount of computation. Note that if $\mathbf{A}$ is of type $\rho$ and belongs to a residually small variety then we will decide in a finite amount of time whether or not $\mathbf{A}$ generates an injectively complete variety, although if it does not belong to a residually small variety we will remain forever undecided.

It seems likely that there is an algorithm for testing if a particular finite algebra of finite type generates a variety with CEP+RS. If we have an algorithm to determine if $\mathrm{A}$ generates a CEP variety, then the method described after Theorem 2.2 will not terminate if $\mathbf{A}$ generates a residually large CEP variety. We pose the following:

Problem. Is there a recursive function $f \varepsilon \omega^{\omega}$ such that for any finite algebra generating a CEP + RS variety it must be that $\mathcal{V}(\mathbf{A})$ is residually $<f(|\mathbf{A}|)$ ?

Emil Kiss has shown that a (possibly non-recursive) function with this property does exist. If such a recursive function were known, then we could use the procedure 
described earlier for $\mathbf{A}$ and terminate the procedure after the $f(|\mathbf{A}|)^{\text {th }}$ step by rejecting $\mathbf{A}$ if we had not already shown that $\mathcal{V}(\mathbf{A})$ has CEP $+\mathrm{RS}$. On the other hand, if $\mathbf{A}$ is shown to generate a CEP+RS variety, then we would apply Theorem 2.5 (c) to check whether A generates a variety with AP. Therefore, if we had an algorithm to test if $\mathcal{V}(\mathrm{A})$ has the CEP and knew such a recursive function $f$, then we would have an algorithm to test if $\mathcal{V}(\mathbf{A})$ has enough injectives.

For distributive varieties, a suitable recursive function is $f_{D}(n)=n+1$. For modular varieties such a function is $f_{M}(n)=n^{\lg n}$ where $\lg n=\log _{2} n$. [ $f_{D}$ works for distributive varieties by Jónsson's Lemma. A proof that $f_{M}$ works for modular varieties can be found in [9].] (On the other hand, the functions $\tilde{f}_{D}(n)=n$ and $\tilde{f}_{M}(n)=$ $n^{1 / 4}(\lg n)$ are too small for infinitely many values of $n$.)

Some work has been done toward understanding which locally finite varieties have the CEP. See [10] and [13], for example. Still, we are a long way from knowing an algorithm which decides if a finite algebra of finite type generates a variety with the CEP.

\section{Congruence Modular Varieties}

For modular varieties many authors have linked commutator conditions to the properties we are considering: RS [6], CEP [11], AP+RS [3] and AP+RS [8]. These results enable us to provide an algorithm to test whether a finite algebra generates an injectively complete modular variety. The relevant commutator conditions are:

$$
\begin{array}{ll}
\text { C1: } & {[x \cdot y, y]=x \cdot[y, y]} \\
\text { C2: } & {[x, y]=x \cdot y \cdot[1,1]} \\
\text { C3: } & {[x, y]=x \cdot y} \\
\text { R: } & \text { If } B \leqslant A, \text { then }\left[1_{\mathbf{B}}, 1_{B}\right]=\left.\left[1_{\mathbf{A}}, 1_{\mathbf{A}}\right]\right|_{\mathbf{B}} .
\end{array}
$$

What is most important for us are the following results.

THEOREM 3.1. [6] A finitely generated, congruence modular variety $\mathcal{V}$ is RS if and only if it contains only finitely many non-isomorphic subdirectly irreducible algbras, all finite, if and only if $\mathcal{V} \vDash$ con $\mathrm{C} 1$.

THEOREM 3.2. A finitely generated, congruence modular variety $\mathcal{V}$ has the CEP if and only if $\mathcal{V} \models$ con $\mathrm{C} 2+\mathrm{R}$ and every $\mathrm{SI}$ in $\mathcal{V}$ has the CEP.

Proof: This follows immediately from Theorems 2.2 and 2.5 in part II of [11]. [

Theorem 3.3. [7] If $\mathbf{A}$ is finite and $\mathcal{V}=\mathcal{V}(\mathbf{A})$ is congruence modular, then $\mathcal{V} \models{ }_{\text {con }} \mathrm{C} i$ for $i=1,2$, or 3 if and only if $\mathrm{A}$ satisfies $\mathrm{Ci}$ hereditarily. $\mathcal{V} \models$ con $\mathrm{C} 3$ if and only if $\mathcal{V}$ is congruence distributive. 
THEOREM 3.4. If $\mathbf{A}$ is finite and $\mathcal{V}=\mathcal{V}(\mathbf{A})$ is congruence modular, then $\mathcal{V} \models$ con $\mathrm{C} 2+\mathrm{R}$ if and only if $\mathrm{A}$ satisfies $\mathrm{C} 2+\mathrm{R}$ hereditarily.

Proof: From Theorem 3.3, $\mathcal{V}$ will satisfy C2 if A satisfies it hereditarily. It follows from Theorem 4.3 of part I of [11] that all that is left to verify is that the (nonabelian) SIs of $\mathcal{V}$ satisfy $\mathrm{R}$. By Theorems 8.3 and 10.1 of [7], the nonabelian SIs are members of $\mathbf{H S}(\mathbf{A})$. We have assumed that $\mathbf{S}(\mathbf{A}) \models_{\text {con }} \mathrm{R}$. If we can show that $\mathrm{R}$ is preserved by onto homomorphisms we will be done.

Assume that $\mathbf{B}$ satisfies $\mathbf{R}$, that $f: \mathbf{B} \rightarrow \mathbf{B}^{\prime}$ is onto and that $\mathbf{B}^{\prime}$ fails to satisfy $\mathrm{R}$. There is a subalgebra $\mathbf{C}^{\prime} \leqslant \mathbf{B}^{\prime}$ such that $\left[1_{\mathbf{C}}^{\prime}, 1_{\mathbf{C}}^{\prime}\right]<\left[1_{\mathbf{B}}^{\prime}, 1_{\mathbf{B}}^{\prime}\right]_{\mathbf{C}}^{\prime}$. Let $\mathbf{C}=f^{-1}\left(\mathbf{C}^{\prime}\right)$ and let $\psi$ denote the kernel of $f$. We've assumed that $\left[1_{\mathbf{C}}, 1_{\mathbf{C}}\right]=\left.\left[\mathbf{1}_{\mathbf{B}}, 1_{\mathbf{B}}\right]\right|_{\mathbf{C}}$. Using the failure of $\mathrm{R}$ in $\mathbf{C}^{\prime}$ and the homomorphism property of the commutator we get

$$
\left(\left.\psi\right|_{\mathbf{C}}+\left.\left[\mathbf{1}_{\mathbf{B}}, \mathbf{1}_{\mathbf{B}}\right]\right|_{\mathbf{C}}\right)<\left.\left(\psi+\left[\mathbf{1}_{\mathbf{B}}, \mathbf{1}_{\mathbf{B}}\right]\right)\right|_{\mathbf{C}}
$$

By Theorem 6.3 of $[7], \psi$ permutes with $\left[1_{\mathbf{B}}, 1_{\mathbf{B}}\right]$. Now choose $\left.(x, y) \varepsilon\left(\psi+\left[1_{\mathbf{B}}, 1_{\mathbf{B}}\right]\right)\right|_{\mathbf{C}} \backslash$ $\left(\left.\psi\right|_{\mathbf{C}}+\left.\left[1_{\mathbf{B}}, 1_{\mathbf{B}}\right]\right|_{\mathbf{C}}\right)$. We have $x \psi z[1,1] y$. We know that $(x, z) \varepsilon \psi$, that $x \in \mathbf{C}$ and that $\mathbf{C}=f^{-1}\left(\mathbf{C}^{\prime}\right)$. From this we may conclude that $z \varepsilon \mathbf{C}$. Hence, $\left.\left.x \psi\right|_{\mathbf{C}} z[1,1]\right|_{\mathbf{C}}, y$. But this contradicts the fact that $\left.(x, y) \notin \psi\right|_{\mathbf{C}}+\left.\left[\mathbf{1}_{\mathbf{B}}, \mathbf{1}_{\mathbf{B}}\right]\right|_{\mathbf{C}}$. This finishes the proof.

Now let us record a few easy facts about injectivity in congruence modular varieties.

LEMMA 3.5. Let $\mathcal{V}$ be a finitely generated, congruence modular variety that has the CEP. $\mathcal{V}$ is residually small.

Proof: $\mathrm{CM}+\mathrm{CEP}$ implies that $\mathcal{V}$ satisfies the commutator congruence condition $\mathrm{C} 2$ by Theorem 3.2, which in turn implies that $\mathcal{V}$ satisfies $\mathrm{C} 1$. Any finitely generated modular variety that satisfies $\mathrm{C} 1$ as a congruence identity is residually small by Theorem 3.1.

PROPOSITION 3.6. Let $\mathcal{A}$ be a modular abelian variety. Then any amalgam $\left(\mathbf{A} ; f_{1}, \mathbf{B} ; g_{1}, \mathbf{C}\right)$ can be completed in $\mathcal{A}$.

Proof: $\mathcal{A}$ has a Malcev term $p(x, y, z)$ that commutes with all the term operations. Let $\mathrm{D}=(\mathbf{B} \times \mathbf{A} \times \mathbf{C}) / \theta$ where $\theta$ is the congruence defined by $\left((b, a, c),\left(b^{\prime}, a^{\prime}, c^{\prime}\right)\right)$ $\varepsilon \theta$ if and only if

$$
p^{\mathbf{B}}\left(b, b^{\prime}, a^{\prime}\right)=\mu \varepsilon \mathbf{A}, p^{\mathbf{C}}\left(c, c^{\prime}, a^{\prime}\right)=\nu \varepsilon \mathbf{A}, \text { and } p^{\mathbf{A}}(\mu, a, \nu)=a^{\prime} .
$$

(For ease of notation we are treating $f_{1}$ and $g_{1}$ as inclusions.) Let $f_{2}: \mathbf{B} \rightarrow \mathbf{D}:$ $b \mapsto(b, a, a) / \theta$ and let $g_{2}: \mathbf{C} \rightarrow \mathbf{D}: c \mapsto(a, a, c) / \theta$. Then $\left(f_{2} ; g_{2} ; \mathbf{D}\right)$ is a completing triple. The necessary verifications that $\theta$ is a congruence and that $f_{2}$ and $g_{2}$ are 1-1 homomorphisms are easy. 
COROllary 3.7. Modular abelian varieties are injectively complete.

Proof: Abelian varieties have RS+CEP as is easily seen from their relationship with varieties of modules as delineated in Chapter 9 of [7]. They have AP by the previous proposition.

Recall that we do not admit the empty algebra. This is essential in Proposition 3.6 and its corollary or else, by taking $\mathbf{A}=\phi$, the amalgamation property translates into the joint embedding property which does not hold for arbitrary modular abelian varieties. For example, let $\mathbf{A}=<\{0,1\}, p, s>$ where $p(x, y, z)=x+y+z(\bmod 2)$ and $s$ is the unary operation that switches 0 and $1 . \mathcal{V}(\mathbf{A})$ is a modular abelian variety in which every linear algebra has only linear subalgebras and (of course) every nonlinear algebra has only nonlinear subalgebras. Hence it is impossible to find an algebra in $\mathcal{V}(\mathbf{A})$ that jointly embeds a linear algebra and a nonlinear algebra.

Proposition 3.8. Let $\mathcal{V}=\mathcal{V}(\mathbf{A})$ be a modular variety generated by the finite algebra $\mathrm{A}$. Assume that $\mathcal{V} \models{ }_{\text {con }} \mathrm{R}$ and let $\mathcal{A} \subseteq \mathcal{V}$ be the subvariety of abelian algebras in $\mathcal{V}$. Then $\mathcal{A}=\operatorname{HSP}(\mathbf{A} /[1,1])$.

Proof: The free algebra $F_{\mathcal{V}}(n)$ is isomorphic to the subalgebra of $\mathbf{A}^{\mathbf{A}^{n}}$ that is generated by the $n$ projections. $F_{\mathcal{A}}(n)=F_{\mathcal{V}}(n) /[1,1]$ which, by $\mathrm{R}$ and by the fact that the commutator behaves well under finite products, is isomorphic to the subalgebra of $\left(\mathbf{A}^{\mathbf{A}^{n}}\right) /[1,1]=(\mathbf{A} /[1,1])^{\mathbf{A}^{n}}$ that is generated by the projections. But this is isomorphic to the $n$-generated free algebra in $\operatorname{HSP}(\mathbf{A} /[1,1])$. Consequently, $\mathcal{A}$ and $\operatorname{HSP}(\mathbf{A} /[1,1])$ satisfy the same equations and must be equal.

A consequence of the last result is that it is fairly easy to locate the subdirectly irreducible algebras in a finitely generated, congruence modular variety that satisfies $\mathrm{C} 2$ and $\mathrm{R}$.

COROLlaRY 3.9. If $\mathbf{A}$ is a finite member of a congruence modular variety and $\mathbf{A} \models_{\text {con }} \mathrm{C} 2+\mathrm{R}$ hereditarily, then $\mathcal{V}=\mathcal{V}(\mathbf{A}) \models_{\text {con }} \mathrm{C} 2+\mathrm{R}$ and

$$
\mathrm{SI}(\mathcal{V}) \subseteq \text { HS }(\mathbf{A}) \cup \operatorname{SI}(\mathcal{V}(\mathbf{A} /[1,1]))
$$

Proof: If $\mathbf{S}(\mathrm{A}) \models_{\text {con }} \mathrm{C} 2+\mathrm{R}$ then $\mathcal{V}$ does by Theorem 8.3 By Theorems 8.3 and 10.1 of [7] the nonabelian SIs of $\mathcal{V}$ are in HS(A). The result of Proposition 3.8 shows that the abelian SIs are in $\operatorname{SI}(\mathcal{V}(\mathbf{A} /[1,1]))$ and this finishes the proof.

The next few results explore some less obvious facts about injectives in modular varieties.

Proposition 3.10. If $\mathcal{V}$ is congruence modular and $\mathbf{A} \times \mathbf{B} \in \mathcal{V}$ is injective in $\mathcal{V}$, then $\mathbf{A}$ is injective in $\mathcal{V}$. 
Proof: Suppose that we have a diagram:

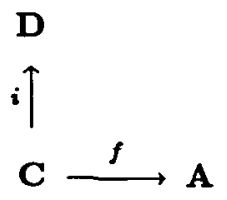

where $i$ is 1-1. By taking products with $\mathrm{B}$ we get another diagram:

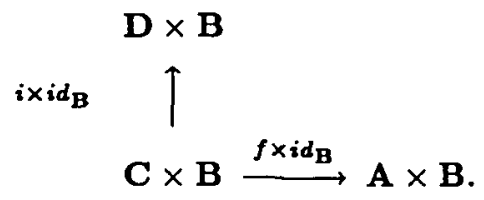

$\mathbf{A} \times \mathbf{B}$ is injective, so there is an $\hat{f}: \mathbf{D} \times \mathbf{B} \longrightarrow \mathbf{A} \times \mathbf{B}$ such that $\hat{f}_{\circ}\left(i \times i d_{\mathbf{B}}\right)=f \times i d_{\mathbf{B}}$. This leads to a homomorphism

$$
g=\pi_{0}^{\mathbf{A}} \times \mathbf{B} \circ \hat{f}: \mathbf{D} \times \mathbf{B} \stackrel{\widehat{f}}{\longrightarrow} \mathbf{A} \times \mathbf{B} \stackrel{\pi_{0}}{\longrightarrow} \mathbf{A}
$$

by composing $\widehat{f}$ with the projection onto $\mathbf{A}$. If $\theta=\operatorname{ker} g,\left.\operatorname{then} \theta\right|_{\mathbf{C} \times \mathbf{B}} \supseteq \eta_{0}^{\mathbf{C} \times \mathbf{B}}$. Therefore, if $(u, v),(x, y) \varepsilon \mathbf{D} \times \mathbf{B}$ and $c \varepsilon \mathbf{C}$, then $(u, v) \eta_{\mathbf{1}}^{\mathbf{D} \times \mathbf{B}}(i(c), v) \boldsymbol{\theta} \cdot \eta_{0}^{\mathbf{D} \times \mathbf{B}}(i(c), y) \eta_{1}^{\mathbf{D} \times \mathbf{B}}(x, y)$ This shows that $\theta \cdot \eta_{0}+\eta_{1}=1$. Of course, $\theta \cdot \eta_{0} \cdot \eta_{1}=0$. Hence, if $\theta \cdot \eta_{0}<\eta_{0}$, then the congruences $0, \theta \cdot \eta_{0}, \eta_{0}, \eta_{1}$ and 1 would form a pentagon, contradicting our modularity hypothesis. We conclude that $\theta \geqslant \eta_{0}$. This means that $g$ factors as

$$
g=\widehat{g} \circ \pi_{0}^{\mathrm{D}} \times \mathrm{B}: \mathrm{D} \times \mathrm{B} \stackrel{\pi_{0}}{\longrightarrow} \mathrm{D} \stackrel{\widehat{g}}{\longrightarrow} \mathrm{A} .
$$

An easy check verifies that $\widehat{g} \circ i=f$ and we are done.

We have just shown that, in a modular variety, a direct product of two algebras is injective only if each factor is. By letting $\mathbf{B}$ represent a direct product of other algebras in our proof, we see that this result holds for arbitrary direct products. It is well-known (and easy to prove) that, in any category, an arbitrary direct product of injective algebras is injective, so the converse of Proposition 3.10 is also true.

Essentially the same proof that we have given shows that, in a modular variety, if an arbitrary direct product of algebras is an absolute retract then each factor must be. Simply replace $\mathbf{C}$ by $\mathbf{A}$ and replace $f$ by $i d_{\mathbf{A}}$ in our argument. It is not true that the converse of this claim is true: there are modular varieties in which a direct product of absolute retracts is not an absolute retract (although in any variety with the CEP a direct product of absolute retracts is again an absolute retract). 
LеммA 3.11. Assume that $\mathcal{V}$ is congruence modular and that $\mathrm{B} \varepsilon \mathcal{V}$. Suppose that $\left\{\alpha_{i} \mid i \varepsilon I\right\}$ is a nonempty set of atomic congruences contained in the centre of $B$ and that $A$ is a subalgebra of $B$ such that $\left.\alpha_{i}\right|_{A}>0_{A}$ for all $i$. If $\alpha$ and $\alpha^{\prime}$ are congruences of $\mathrm{B}$ such that $\alpha \leqslant \bigvee_{i \in I} \alpha_{i}$ and $\alpha \notin \alpha^{\prime}$, then $\left.\left.\alpha\right|_{A} \nless \alpha^{\prime}\right|_{A}$. In particular, if $\alpha>0_{\mathbf{B}}$ then $\left.\alpha\right|_{\mathbf{A}}>0_{\mathbf{A}}$.

Proof: Let $\beta=\bigvee_{i e I} \alpha_{i}$. Choose an element of $\mathbf{A}$ and call it 0 . Let $M(\beta, 0)$ be the $\beta$-class of $B$ containing 0 . The results of Chapter 9 of [7] show that $M(\beta, 0)$ is the universe of a module over the ring $\mathbf{R}=\mathbf{R}(\mathcal{V}, 1,1)$ (we choose 0 as our only constant). Since $[1, \beta]=\bigvee_{i e I}\left[1, \alpha_{i}\right]=0$, Theorem 9.9 of $[7]$ shows that the lattice of submodules of $\mathbf{R} \mathbf{M}(\beta, 0)$ is naturally isomorphic to the interval $I[0, \beta]$ in Con $\mathbf{B}$. Since $\left.\alpha_{i}\right|_{\mathbf{A}}>0_{\mathbf{A}}$, there exist $u_{i}, v_{i} \varepsilon \mathrm{A}$ such that $\alpha_{i}=\mathrm{Cg}_{\mathrm{B}}\left(u_{i}, v_{i}\right)$. Let $x_{i}=d\left(u_{i}, v_{i}, 0\right) \varepsilon \mathrm{A}$ where $d$ is a modular difference term for $\mathcal{V}$. Note that $x_{i} \neq 0$, since otherwise $d\left(u_{i}, v_{i}, 0\right)=$ $d\left(v_{i}, v_{i}, 0\right)$ and the $1, \beta$-term condition in $\mathbf{B}$ forces $u_{i}=d\left(u_{i}, 0,0\right)=d\left(v_{i}, 0,0\right)=v_{i}$ which is false. Hence $\left(x_{i}, 0\right)=\left(d\left(u_{i}, v_{i}, 0\right), d\left(v_{i}, v_{i}, 0\right)\right) \varepsilon \alpha_{i} \backslash 0$ and this implies that $\alpha_{i}=\mathrm{Cg}_{\mathbf{B}}\left(x_{i}, 0\right)$. By the same argument, if $(y, z) \varepsilon \alpha \backslash \alpha^{\prime}$, then $(w, 0) \varepsilon \alpha \backslash \alpha^{\prime}$ where $w=d(y, z, 0)$.

Since $\alpha \leqslant \bigvee_{i \varepsilon I} \alpha_{i}$, it follows from the natural correspondence between submodules of $\mathbf{R} \mathbf{M}(\beta, 0)$ and congruences that are below $\beta$ that $w \varepsilon \sum \mathbf{R}_{x_{i}}$. In other words, there are elements $m_{i_{1}}, \ldots, m_{i_{n}}$ in $R$ such that $w=m_{i_{1}} x_{i_{1}}+\cdots+m_{i_{n}} x_{i_{n}}$. In $B$ this means that there is an $(n+1)$-ary term of $\mathcal{V}, f$, such that $w=f\left(x_{i_{1}}, \ldots, x_{i_{n}}, 0\right)$. But all of the elements $0, x_{i_{1}}, \ldots, x_{i_{n}}$ are in $\mathbf{A}$, so we get that $w \varepsilon \mathbf{A}$. Thus, $\left.\left.(w, 0) \varepsilon \alpha\right|_{\mathbf{A}} \backslash \alpha^{\prime}\right|_{\mathbf{A}}$. $\square$

ThEOREM 3.12. Assume that $\mathcal{V}$ is congruence modular and that $\mathbf{A} \varepsilon \mathcal{V}$ is injective. If Con $\mathbf{A}$ satisfies the descending chain condition (DCC), then $\mathbf{A}$ is a finite direct product of SI injectives.

Proof: We begin with a claim.

ClaIM. There is a subdirect representation $A \leqslant \prod_{i=0}^{n} A_{i}$ where each $A_{i}$ is $S I$ and $\left.\left(\eta_{0} \cdots \eta_{i}^{*} \cdots \eta_{n}\right)\right|_{A}>0$ for all $i$. (Here $\eta_{i}^{*}$ denotes the unique upper cover of the projection congruence $\eta_{i}$.)

We will write $\tilde{\eta}_{i}$ to denote the restriction of $\eta_{i}$ to $\mathbf{A}$. Now, our claim is equivalent to the assertion that $\mathbf{0}_{\mathbf{A}}$ is decomposable as a finite meet of strictly meet-irreducible congruences $0_{A}=\tilde{\eta}_{0} \cdots \tilde{\eta}_{n}$ such that $\tilde{\eta}_{0} \cdots \tilde{\eta}_{i}^{*} \cdots \tilde{\eta}_{n}>0$ for each $i$.

To prove this claim, first let $S$ be the set of congruences on $\mathbf{A}$ which are finite meets of strictly meet-irreducible congruences on $\mathbf{A}$. Since Con $\mathbf{A}$ has DCC, $S$ has at least one minimal element which we will call $\delta$. If $\eta$ is a strictly meet-irreducible congruence, then the minimality of $\delta$ forces $\eta \cdot \delta \geqslant \delta$ and so $\eta \geqslant \delta$. $\eta$ was arbitrary so 
$\delta$ must be the zero congruence. This shows that $0_{\mathbf{A}}$ has a finite decomposition: $0_{\mathbf{A}}=$ $\tilde{\eta}_{0} \cdots \tilde{\eta}_{n}$. Assume that $n$ is the least natural number for which such a decomposition exists. Let $\vec{\eta}_{i}=\tilde{\eta}_{0} \cdots \tilde{\eta}_{i-1} \tilde{\eta}_{i+1} \cdots \tilde{\eta}_{n}$. By the minimality of $n, \vec{\eta}_{i}>0$ for all $i$. Now, $\left(\widetilde{\eta}_{i}+\tilde{\eta}_{i}^{\prime}\right) / \tilde{\eta}_{i} \searrow \tilde{\eta}_{i} / 0$ so $I\left[0, \tilde{\eta}_{i}^{\prime}\right]$ is a monolithic interval with unique atom $\tilde{\eta}_{i}^{*} \cdot \widetilde{\eta}_{i}=$ $\tilde{\eta}_{0} \cdots \tilde{\eta}_{i}^{*} \cdots \tilde{\eta}_{n}$. Hence our decomposition $\mathbf{0}_{\mathbf{A}}=\tilde{\eta}_{0} \cdots \tilde{\eta}_{\boldsymbol{n}}$ has the desired property that $\tilde{\eta}_{0} \cdots \tilde{\eta}_{i}^{*} \cdots \tilde{\eta}_{n}>0$ for each $i$. This establishes our claim.

We will establish that the subdirect representation obtained above is actually a direct product representation. To do this it suffices to show that the embedding $A \leqslant \prod_{i=0}^{n} \mathbf{A}_{i}$ is essential, since $\mathbf{A}$ is injective and consequently has no proper essential extensions.

It is straightforward to show that since Con $\mathbf{A}$ has the descending chain condition that Con $\prod_{i=0}^{n} \mathbf{A}_{i}$ also has DCC. Therefore, Con $\prod_{i=0}^{n} \mathbf{A}_{i}$ is an atomic lattice. In order to prove that $\mathbf{A} \leqslant \prod_{i=0}^{n} \mathbf{A}_{i}$ is essential, we only need to rule out the possibility that there is an atom $\alpha$ of Con $\prod_{i=0}^{n} A_{i}$ with the property that $\left.\alpha\right|_{A}=0$.

Let $\alpha_{i}$ denote the atom $\eta_{i}^{*} \cdot \eta_{i}^{\prime}=\eta_{0} \cdots \eta_{i}^{*} \cdots \eta_{n}$ of Con $\prod_{i=0}^{n} \mathbf{A}_{i}$. Now choose an

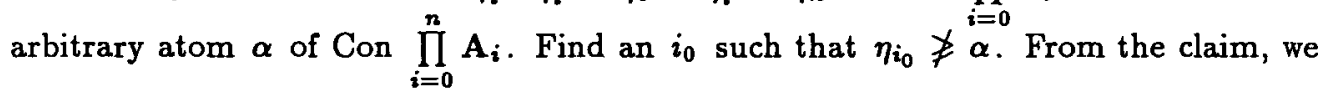
see that the choice of the $\eta_{i}$ was such that $\left.\alpha_{i}\right|_{A}>0$ for all $i$, so if $\alpha=\alpha_{i_{0}}$ we are done. Assume that $\alpha \neq \alpha_{i_{0}}$. Now, $I\left[0, \eta_{i_{0}}^{\prime}\right]$ is a monolithic interval with unique atom $\alpha_{i_{0}}$. We conclude that $\eta_{i_{0}}^{\prime} \supsetneqq \alpha$. It follows that

$$
0=\eta_{i_{0}} \cdot \alpha+\eta_{i_{0}}^{\prime} \cdot \alpha \supseteq\left[\eta_{i_{0}}, \alpha\right]+\left[\eta_{i_{0}}^{\prime}, \alpha\right]=[1, \alpha]
$$

so $\alpha$ is a central congruence of $\prod_{i=0}^{n} \mathbf{A}_{i}$. By reordering the $\eta_{i}$ if necessary, we may assume that there is a $k \leqslant n$ such that $\eta_{i} ¥ \alpha$ for $i \leqslant k$, but $\eta_{i} \geqslant \alpha$ for $i>k$. Of course, $\eta_{i}^{*} \geqslant \alpha$ for all $i$, so $\alpha \leqslant \eta_{0}^{*} \cdots \eta_{k}^{*} \cdot \eta_{k+1} \cdots \eta_{n}=\beta$. Notice that $\beta=\bigvee_{i=0}^{k} \alpha_{i}$. Notice also that for $i \leqslant k, \alpha / 0 \nearrow \eta_{i}^{*} / \eta_{i} \searrow \alpha_{i} / 0$. This condition shows that $\alpha_{j}$ is central for $j \leqslant k$. Now we are in the situation of Lemma 3.11. That is, $\alpha$ is contained in the join of central atoms, each of which restricts nontrivially to $A$, and $\alpha$ is not contained in the zero congruence of $\prod_{i=0}^{n} \mathbf{A}_{i}$. Hence, $\left.\alpha\right|_{\mathbf{A}}$ is not 0 . This rules out the possibility that Con $\prod_{i=0}^{n} A_{i}$ has an atom that restricts to the zero congruence on $A$. Hence $A \cong \prod_{i=0}^{n} A_{i}$. By Proposition 3.10 each factor is injective and this establishes the theorem.

The proof that we have just given shows that, in a modular variety $\mathcal{V}$, every algebra whose congruence lattice has DCC can be essentially embedded into a finite direct product of SI algebras (where each factor has DCC on congruences). One consequence 
of this is that any absolute retract in $\mathcal{V}$ whose congruence lattice has DCC is isomorphic to a finite direct product of SI absolute retracts. This kind of factorisation theorem for absolute retracts or for injectives does not hold for varieties in general. For example, if $\mathcal{G}$ is a variety of $G$-sets, then the $G$-set with three trivial orbits is injective but it has no direct product decomposition into SI algebras. If $\mathcal{S}$ is the variety of semilattices, then the three-element chain is injective in $\mathcal{S}$ but it has no such decomposition. We make the following conjecture:

CONJECTURE. If $\mathcal{V}$ is a locally finite variety with enough injectives, then every finite injective algebra is isomorphic to a direct product of injective SIs if and only if $\mathcal{V}$ is congruence modular.

A proof of this conjecture for any $\mathcal{V}$ which satisfies a nontrivial idempotent Mal'cev condition can be found in [10].

The decomposition of injective algebras described in Theorem 3.12, along with other known theorems of this sort lead us to pose two problems:

Problem. Can one describe the structure of the injective algebras in an arbitrary congruence modular variety which are directly indecomposable?

and

PROBLEM. Is there some kind of boolean construction which one can use to build arbitrary injective algebras in a congruence modular variety from indecomposable injective algebras?

Kollár's Theorem states that if $\mathcal{K}=\operatorname{HSSI}(\mathcal{V})$ where $\mathcal{V}$ is a a finitely generated, congruence distributive variety, then $\mathcal{V}$ has enough injectives if and only if every maximal SI in $\mathcal{V}$ is *-injective over $\mathcal{K}$ and every retract of a maximal $\mathrm{SI}$ is isomorphic to a direct product of SI algebras that are ${ }^{*}$-injective over $\mathcal{K}$. Before we prove Theorem 1.1 , which extends Kollár's Theorem, let us consider an example which shows that Kollár's Theorem for congruence distributive varieties cannot be extended "intact" to include congruence modular varieties. That is, Kollár's Theorem becomes false if we simply replace the word "distributive" with the word "modular".

EXAMPLE 3.13: Let $\mathbf{A}$ be the symmetric group on 3 letters. It is a fairly easy exercise to show that the only non-trivial SIs in $\mathcal{V}(\mathbf{A})$ are $\mathbf{A}$, the 2-element group $\mathbf{Z}_{2}$ and the 3-element group $Z_{3}$. The non-isomorphic groups in $\mathcal{K}=\mathbf{H S S I}(\mathcal{V}(\mathbf{A}))$ are just these three groups and the trivial group. The only maximal SI is $A$. $\mathbf{Z}_{3}$ is the only member of $\mathcal{K}$ which is not *-injective and it is also the only one which is not a retract of A. Except for congruence distributivity, the conditions of Kollár's Theorem hold for this variety. However, $\mathbf{A}$ does not satisfy $\mathrm{C} 2$ or $\mathbf{R}$, so $\mathcal{V}(\mathbf{A})$ does not have the CEP. $\mathcal{V}(\mathbf{A})$ cannot have enough injectives. (This variety is residually small and does not have the CEP so, by one of the principal result of [8], it does not have AP.) 
Now we are in a position to prove Theorem 1.1.

THEOREM 1.1. Let $\mathcal{V}$ be a finitely generated, congruence modular variety. Let $\mathcal{K}=\operatorname{HSSI}(\mathcal{V})$. Then $\mathcal{V}$ has enough injectives if and only if:

(a) $\mathcal{K} \models_{\text {con }} \mathrm{C} 2+\mathrm{R}$,

(b) Each maximal SI is *-injective over $\mathcal{K}$ and

(c) Every retract of a maximal SI is isomorphic to a direct product of SI algebras that are ${ }^{*}$-injective over $\mathcal{K}$.

ProOF: First, we assume that $\mathcal{V}$ has enough injectives and we show that (a), (b) and (c) hold. Every modular variety with the $\mathrm{CEP}$ satisfies $\mathrm{C} 2+\mathrm{R}$, so $\mathcal{K} \vDash$ con $\mathrm{C} 2+\mathrm{R}$. Now, any proper essential extension of a subdirectly irreducible algebra is $\mathrm{SI}$, so maximal SIs have no proper essential extensions. This implies that any maximal SI is an absolute retract in $\mathcal{V}$. Since $\mathcal{V}$ has enough injectives, maximal SIs must be injective in $\mathcal{V}$. A fortiori, any maximal SI is *-injective over $\mathcal{K}$. What remains to show is that if $\mathbf{R}$ is a retract of a maximal SI, then $\mathbf{R}$ factors as a direct product of SIs that are ${ }^{*}$-injective over $\mathcal{K}$. Such an $\mathbf{R}$ must be finite (since all the SIs in $\mathcal{V}$ are finite) and injective (since retracts of injective algebras are injective). Proposition 3.12 applies and shows that $\mathbf{R}$ does have such a factorisation.

Now assume that the conditions (a), (b) and (c) hold. Since $\mathcal{K} \models_{\text {con }} \mathrm{C} 2$ and $\mathcal{K}$ contains all the SIs, $\mathcal{V} \models_{\text {con }} \mathrm{C} 2 . \mathcal{V}$ is finitely generated and satisfies $\mathrm{C} 2$ so it satisfies the weaker condition C1. By Theorem 3.1, $\mathcal{V}$ has only finitely many isomorphism types of SIs, all finite. Hence $\mathcal{V}$ is RS. Now $\mathcal{K}$ is closed under ultraproducts (since it contains only finitely many isomorphism types of algebras, all finite) and $\mathcal{K}$ contains all SIs. Since $\mathcal{K} \models_{\text {con }} \mathrm{R}$ it follows from Theorem 2.2 of part II of [11] that $\mathcal{V} \models_{\text {con }} \mathrm{R}$. By Theorem 3.2 $\mathcal{V}$ has the CEP if and only if each SI in $\mathcal{V}$ has the CEP. To verify that this is so let $\mathbf{A} \in \operatorname{SI}(\mathcal{V})$, let $\mathbf{B}$ be a subalgebra of $\mathbf{A}$ and let $\theta$ be a strictly meet-irreducible congruence of $B$. Now, $B / \theta$ is SI and we can embed it into a maximal SI, C:

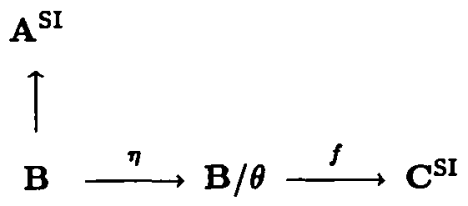

Since $\mathbf{A}, \mathbf{B}$ and $\mathbf{C} \varepsilon \mathcal{K}$ and $\mathbf{C}$ is *-injective over $\mathcal{K}$ we can extend $f \circ \eta$ to $\hat{f}$ : $\mathbf{A} \rightarrow \mathbf{C}$, thereby extending $\theta$ to ker $\hat{f}$. This shows that every strictly meet-irreducible congruence on $\mathbf{B}$ can be extended to $\mathbf{A}$. By representing an arbitrary congruence as a meet of strictly meet-irreducible congruences we see that arbitrary congruences on $B$ can be extended to $\mathbf{A}$. Thus, $\mathbf{A}$ has the CEP.

To finish, we only need to verify condition (c) of Theorem 2.5. Note that (c)(i) holds by hypothesis. Suppose that $\mathbf{R}$ is a retract of a maximal SI. By our hypothesis, 


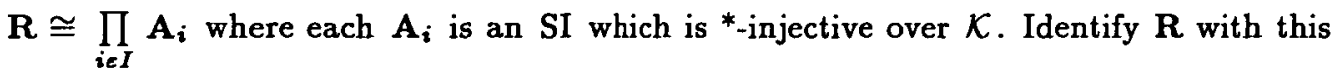
product and let $\left\{\eta_{i} \mid i \in I\right\}$ be the kernels of the coordinate projections. The $\eta_{i}$ are strictly meet-irreducible and they separate the points of $\mathbf{R}$. Further, if $f: \mathbf{R} / \boldsymbol{\eta}_{\boldsymbol{i}} \cong \mathbf{A}_{\boldsymbol{i}} \rightarrow$ $\mathbf{B}_{i}$ is a maximal essential extension of $\mathbf{A}_{i}$, then $f$ is an isomorphism. This is because any essential extension of $\mathbf{A}_{i}$ is $\mathrm{SI}$, and therefore in $\mathcal{K}$. But $\mathbf{A}_{i}$ is ${ }_{\text {-injective in } \mathcal{K}}$ which means that $\mathbf{A}_{i}$ is an absolute retract in $\mathcal{K}$; this precludes any proper essential extensions of $\mathbf{A}_{i}$. We may assume that $\mathbf{B}_{i}=\mathbf{A}_{\boldsymbol{i}}$. Any shell $\mathbf{S}$ of $\mathbf{R}$ with respect to the congruences $\left\{\eta_{i} \mid i \in I\right\}$ is an essential extension of $\mathbf{R}$ which can be embedded in $\prod_{i \varepsilon I} \mathbf{B}_{i}=\prod_{i \varepsilon I} \mathbf{A}_{\boldsymbol{i}}=\mathbf{R}$. A cardinality argument already shows that $\mathbf{S}=\mathbf{R}$, so condition (c) holds.

COROLlary 3.14. (J. Kollár [12]) Let $\mathcal{V}$ be a finitely generated, congruence distributive variety. Let $\mathcal{K}=\operatorname{HSSI}(\mathcal{V})$. Then $\mathcal{V}$ has enough injectives if and only if every maximal SI is *-injective over $\mathcal{K}$ and every retract of a maximal SI is isomorphic to a direct product of SI algebras that are ${ }^{*}$-injective over $\mathcal{K}$.

Proof: Congruence distributive varieties are congruence modular and satisfy $\mathrm{C} 2+\mathrm{R}$.

Our phrasing is slightly different from Kollár's. Throughout his paper he uses the word injective to mean what we call *injective.

If we are given a finite algebra $A$ of finite type there is an algorithm to determine if it generates a congruence modular variety: compute all 4-variable terms and check to see if any sequence of terms is a sequence of Day terms for modularity. If it generates a congruence modular variety, it is quite easy to check whether $\mathbf{A}$ satisfies $\mathbf{C 2}+\mathbf{R}$ hereditarily. If not, then $\mathbf{A}$ does not generate an injectively complete variety. On the other hand, if $\mathrm{A}$ does pass this test and $|A|=n$, then $n \lg \mathbf{n}$ bounds the size of the subdirectly irreducible algebras in $\mathcal{V}(\mathbf{A})$. This number clearly bounds the size of the algebras in $\mathcal{K}$. From this one can produce a bound on the number of algebras in $\mathcal{K}$ as well. Clearly there is an effective algorithm to check whether or not the conditions of Theorem 1.1 hold, since these conditions only involve the algebras in $\mathcal{K}$. Thus, there is an algorithm to determine if a finite algebra generates a congruence modular, injectively complete variety.

The conditions on the finite algebra $\mathbf{A}$ which guarantee that $\mathcal{V}(\mathbf{A})$ has enough injectives can be phrased more directly in terms of $\mathbf{A}$ if we add some restrictive hypotheses. First we impose a restriction on the subalgebras of $\mathbf{A}$ and obtain conditions phrasable in terms of the congruences on $A$. Then we impose a restriction on the congruences of $\mathbf{A}$ and get conditions phrasable (for the most part) in terms of subalgebras.

COROLlary 3.15. Let $\mathbf{A}$ be a finite algebra with no non-trivial subalgebras 
which generates a modular variety. $\mathcal{V}=\mathcal{V}(\mathbf{A})$ has enough injectives if and only if $\mathbf{A} \models_{\text {con }}$ C2.

Proof: The forward direction is clear. For the other direction, if $\mathbf{A} \models_{\text {con }}$ C2 then $\mathrm{S}(\mathrm{A}) \models_{\text {con }} \mathrm{C} 2+\mathrm{R}$. We may conclude from Theorem 3.4 that $\mathcal{V} \models_{\text {con }} \mathrm{C} 2+\mathrm{R}$. $\mathcal{K}=\operatorname{HSSI}(\mathcal{V})$ satisfies $\mathrm{C} 2+\mathrm{R}$ as well. This shows that Theorem 1.1(a) holds. Further, the proof of Corollary 3.9 shows that the nonabelian algebras in $\mathcal{K}$ are homomorphic images of $\mathbf{A}$, hence they have no non-trivial subalgebras.

To verify Theorem 1.1(b), assume that $I$ is a maximal SI which is not *-injective over $\mathcal{K}$. We must have a diagram in $\mathcal{K}$

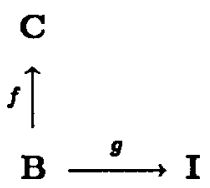

where $f$ is $1-1$ but there is no $\widehat{g}$ such that $g=\widehat{g} \circ f$. Necessarily, $f$ is not an isomorphism and the image of $g$ has more than one element. Since the nonabelian algebras in $\mathcal{K}$ have no non-trivial subalgebras, all three algebras are abelian. But $I$ is a maximal SI in the abelian subvariety of $\mathcal{V}$. This means that $I$ is injective in the abelian subvariety. This contradicts the assumption that no $\widehat{g}$ exists so 1.1 (b) holds.

The only non-trivial retracts of maximal SIs occur in abelian maximal SIs. They must factor into a direct product of SI algebras in $\mathcal{V}$ since they do in the abelian subvariety of $\mathcal{V}$. The factors are injective in the abelian subvariety of $\mathcal{V}$, so they can't fail to be *-injective in $\mathcal{K}$. This is because, as we have noted, the only "interesting" injectivity diagrams involve only abelian algebras.

COROLlary 3.16. Let $\mathbf{A}$ be a finite simple algebra such that $\mathcal{V}=V(\mathbf{A})$ is congruence modular. Then $\mathcal{V}$ is injectively complete if and only if $\mathbf{A}$ is abelian or:

(a) $\mathbf{A}$ is hereditarily simple,

(b) $\mathcal{V}$ is congruence distributive and

(c) every isomorphism between non-trivial subalgebras of $\mathbf{A}$ extends to an automorphism of $\mathbf{A}$.

Proof: By Corollary 3.7, any abelian algebra generates an injectively complete variety so let us assume that $A$ is nonabelian and generates an injectively complete variety. If $\mathbf{A}$ is simple and has the CEP then it is hereditarily simple. $\mathbf{A} /[1,1]$ is trivial so, by Proposition 3.8, $\mathcal{V}$ has no abelian algebras. This means that each subalgebra of $A$ is simple and nonabelian. A must satisfy C3 hereditarily so, by Theorem $3.3, \mathcal{V}$ is congruence distributive. We only need to show that condition (c) holds to conclude the proof of the "only if" part of this corollary. 
Suppose that $\mathbf{B}$ is a non-trivial subalgebra of $\mathbf{A}$ and that $f_{1}$ and $g_{1}$ are two embeddings of $\mathbf{B}$ into $\mathbf{A}$. (B; $\left.f_{1}, \mathbf{A} ; g_{1}, \mathbf{A}\right)$ is an amalgam where both $f_{1}$ and $g_{1}$ are essential. By Lemma 2.3, there is a completing triple $\left(f_{2} ; g_{2} ; \mathbf{D}\right)$ where $g_{2}: \mathbf{A} \rightarrow \mathbf{D}$ is essential. Necessarily, $\mathbf{D}$ is SI and, by (a) and (b) and Jónsson's Lemma, $\mathbf{D}$ is a subalgebra of $A$. By cardinality considerations $f_{2}$ and $g_{2}$ are isomorphisms. Let $\alpha$ denote the automorphism of $\mathbf{A}$ given by $g_{2}^{-1} \circ f_{2}$. We have $g_{1}=\alpha \circ f_{1}$, so these two embeddings of $\mathbf{B}$ differ by an automorphism of $\mathbf{A}$. This shows that condition (c) holds.

Now we assume (a), (b) and (c) hold and we'll show that $\mathcal{V}$ is injectively complete. By (a), (b) and Jónsson's Lemma, $\mathcal{K}=\operatorname{HSSI}(\mathcal{V})$ consists of the subalgebras of $\mathbf{A}$ and trivial algebras. The only maximal SI in $\mathcal{K}$ up to isomorphism is $\mathbf{A}$ and $\mathbf{A}$ has only trivial retracts. By Corollary 3.14 we only need to show that $\mathbf{A}$ is *-injective over $\mathcal{K}$. To verify this, assume that $\mathbf{B}, \mathbf{C} \varepsilon \mathcal{K}$ and

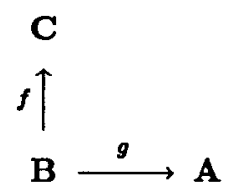

where $f$ is $1-1$. If $g$ is constant, then let $\hat{g}$ be the constant homomorphism that satisfies $\widehat{g} \circ f=g$. If $g$ is nonconstant, then $|C| \geqslant|B|>1$ so $\mathrm{B}, \mathrm{C} \varepsilon \mathrm{S}(\mathrm{A})$ and $g$ is 1-1. Let $i: \mathbf{C} \rightarrow \mathbf{A}$ be the inclusion map. Now $g$ and $i$ of are embeddings of the non-trivial algebra $\mathbf{B}$ into $\mathbf{A}$. By condition (c), there is an automorphism $\alpha$ of $\mathbf{A}$ such that $\alpha \circ i \circ f=g$. Let $\widehat{g}=\alpha \circ i: \mathbf{C} \rightarrow \mathbf{A}$. We have $\widehat{g} \circ f=g$ so we are done.

An immediate consequence of this corollary is the result of Day:

Corollary 3.17. Every primal algebra generates an injectively complete variety.

Proof: Primal algebras are finite, simple, have no non-trivial subalgebras and generate a distributive variety.

Definition: A finite algebra $\mathbf{A}$ is called quasiprimal if it has a ternary term $t$ satisfying:

$$
t^{\mathbf{A}}(x, y, z)=x \text { if } x \neq y \text {, and } t^{\mathbf{A}}(x, y, z)=z \text { if } x=y .
$$

We call $t$ a discriminator term for $\mathbf{A}$.

Pixley [15] has characterised quasiprimal algebras as finite, hereditarily simple algebras that generate an arithmetical variety (that is, one that is both distributive and permutable). In particular, quasiprimal algebras generate $\mathrm{CEP}+\mathrm{RS}$ varieties. Armed with these facts and Corollary 3.16 we get Quackenbush's result:

Corollary 3.19. If $\mathbf{A}$ is quasiprimal then $\mathcal{V}(\mathbf{A})$ has AP if and only if every isomorphism between non-trivial subalgebras of $\mathbf{A}$ extends to an automorphism of $\mathbf{A}$ (that is, A is demi-semi-primal). 
Theorem 3.20. Assume that $\mathcal{V}$ is a locally finite, modular variety that is the join of its minimal subvarieties. Then $\mathcal{V}$ is injectively complete.

Proof: Any locally finite variety has finitely many minimal subvarieties and each one is generated by a strictly simple algebra (a finite, simple algebra that has no nontrivial proper subalgebras). A strictly simple member of a modular variety is abelian or else satisfies C3 (hereditarily). Hence minimal, locally finite, modular varieties are either abelian or distributive. Let $\mathcal{A}$ denote the join of the abelian minimal subvarieties and let $\mathcal{D}$ denote the join of the distributive ones. $\mathcal{A}$ is abelian and $\mathcal{D}$ is distributive, so $\mathcal{V}=\mathcal{A} \otimes \mathcal{D}$. It is easy to see that $\mathcal{K}=\operatorname{HSSI}(\mathcal{V})$ consists of these strictly simple algebras and trivial algebras, so the only homomorphisms in $\mathcal{K}$ which are not isomorphisms are the constant homomorphisms. Every member of $\mathcal{K}$ is simple or trivial and is *-injective over $\mathcal{K}$. Clearly the conditions of Theorem 1.1 must hold.

Corollary 3.21. (A. Day [5]) Every minimal, locally finite CD variety is injectively complete. (Actually, Day's result is expressed in terms of $\operatorname{Mod} \Sigma(\mathcal{V})$ and so it looks a little different.)

Outside the modular domain Theorem 3.20 and Corollary 3.21 fail. In [4], Bergman and McKenzie have provided examples of minimal, locally finite varieties that are not residually small. Local finiteness is also an essential hypothesis for us. In [17], Taylor gives an example to show that minimal $C D$ varieties that are not locally finite may be residually large.

\section{REFERENCES}

[1] J.T. Baldwin and J. Berman, 'The number of subdirectly irreducible algebras in a variety', Algebra Universalis 5 (1975), 379-389.

[2] B. Banaschewski, 'Injectivity and essential extensions in equational classes of algebras', Proceedings of the Conference on Universal Algebra (October 1969), in Queen's Papers in Pure and Applied Mathematics 25, (Kingston, Ontario, 1970), pp. 131-147.

[3] C. Bergman and R. McKenzie, 'On the relationship of AP, RS and CEP in congruence modular varieties. II', Proc. Amer. Math. Soc. 103 (1988), 335-343.

[4] C. Bergman and R. McKenzie, 'When a minimal variety is a minimal quasivariety', $J$. Austal. Math. Soc. (to appear).

[5] A. Day, 'Injectivity in equational classes of algebras', Canad. J. Math. 24 (1972), 209-220.

[6] R. Freese and R. McKenzie, 'Residually small varieties with modular congruence lattices', Trans. Amer. Math. Soc. 264 (1981), 419-430.

[7] R. Freese and R. McKenzie, Commutator theory for congruence modular varieties: LMS Lecture Note Series 125 (Cambridge, 1987).

[8] K.A. Kearnes, 'On the relationship between AP, RS and CEP', Proc. Amer. Math. Soc. 4 (1989), 827-839.

[9] K.A. Kearnes, 'Residual bounds in varieties of modules', Algebra Universalis (to appear). 
[10] K.A. Kearnes, 'Type restriction in locally finite varieties with the CEP', Canad. J. Math. (to appear).

[11] E. Kiss, 'Injectivity and related concepts in modular varieties I-II', Bull. Austral. Math. Soc. 32 (1985), 35-53.

[12] J. Kollár, 'Injectivity and congruence extension property in congruence distributive equational classes', Algebra Universalis 10 (1980), 21-26.

[13] R. McKenzie, 'Congruence extension, Hamiltonian and Abelian properties in locally finite varieties' (to appear).

[14] R. McKenzie, G. McNulty and W. Taylor, Algebras, lattices, varieties (Wadsworth Inc. and Brooks/Cole Publishing Co., 1987).

[15] A.F. Pixley, 'The ternary discriminator function in universal algebra', Math. Ann. 191 (1971), 167-180.

[16] R. Quackenbush, 'Structure theory for equational classes generated by quasiprimal algebras', Trans. Amer. Math. Soc. 187 (1974), 127-145.

[17] W. Taylor, 'Residually small varieties', Algebra Universalis 2 (1972), 33-53.

\footnotetext{
Department of Mathematics

Vanderbilt University

Nashville TN 37235

United States of America
} 